\title{
Substorm topology in the ionosphere and magnetosphere during a flux rope event in the magnetotail
}

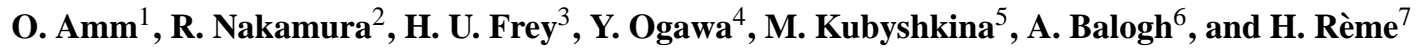 \\ ${ }^{1}$ Finnish Meteorological Institute, Space Physics program, P.O. Box 503, FIN-00101 Helsinki, Finland \\ ${ }^{2}$ Space Research Institute, Austrian Academy of Sciences, Schmiedlstraße 6, 8042 Graz, Austria \\ ${ }^{3}$ University of California, Space Sciences Laboratory, Berkeley, CA 947207450, USA \\ ${ }^{4}$ SolarTerrestrial Environment Laboratory, Nagoya University, Furo-cho, Chikusa-ku, Nagoya, 4648601, Japan \\ ${ }^{5}$ Institute of Physics, University of St. Petersburg, St. Petersburg 198904, Russia \\ ${ }^{6}$ Imperial College London, Space \& Atmospheric Physics Group, The Blackett Laboratory, London SW7 2BW, UK \\ ${ }^{7}$ CESR/CNRS, 9, Avenue du Colonel Roche, 31028 Toulouse Cedex 4, France
}

Received: 25 August 2005 - Revised: 2 December 2005 - Accepted: 8 February 2006 - Published: 23 March 2006

\begin{abstract}
On 13 August 2002, at 23:00 UT, about $10 \mathrm{~min}$ after a substorm intensification, Cluster observes a flux rope in the central magnetotail, followed by a localised fast flow event about one minute later. Associated with the flux rope event, a traveling compression region (TCR) is seen by those Cluster spacecraft which reside in the lobe. In the conjugate ionospheric region in Northern Scandinavia, the MIRACLE network observes the ionospheric equivalent currents, and the electron densities and electric fields are measured by the EISCAT radar along a meridional scanning profile. Further, the auroral evolution is observed with the Wideband Imaging Camera (WIC) on the IMAGE satellite. We compare in detail the substorm evolution as observed in the ionosphere and in the magnetosphere, and examine whether topological correspondences to the flux rope event exist in the ionospheric signatures. The large-scale mapping of both the location and the direction of the flux rope to the ionosphere shows an excellent correspondence to a lens-shaped region of an auroral emission minimum. This region is bracketed by an auroral region equatorward of it which was preexisting to the substorm intensification, and a substorm-related auroral region poleward of it. It is characterised by reduced ionospheric conductances with respect to its environment, and downward field-aligned current (FAC) observed both in the magnetosphere and in the ionosphere. As determined from the ionospheric data, this downward FAC area is moving eastward with a speed of $\sim 2 \mathrm{~km} \mathrm{~s}^{-1}$, in good agreement with the mapped plasma bulk velocity measured at the Cluster
\end{abstract}

Correspondence to: $\mathrm{O} . \mathrm{Amm}$

(olaf.amm@fmi.fi) satellite closest to that area. Further southwestward to this leading downward FAC area, a trailing upward FAC area is observed that moves eastward with the same speed. The direction of the ionospheric electric field permits a current closure between these two FAC areas through the ionosphere. We speculate that these FAC areas may correspond to the ends of the flux rope in its symmetry direction.

Keywords. Ionosphere (Auroral ionosphere; Electric fields and currents) - Magnetospheric physics (Magnetosphereionosphere interactions)

\section{Introduction}

Helical magnetic field structures inside the magnetosphere have played an important role for the understanding of the process of magnetospheric substorms since the early models of Schindler (1974) and Hones (1977), in which such structures, termed "plasmoids", played a major role in transporting the substorm energy antisunwards. The minimum energy state of such helical magnetic field structures is represented by force-free magnetic flux ropes (Priest, 1990). These are elongated magnetic structures in the central axis of which the magnetic field is strongest and points along that axis. With increasing radial distance from that axis the field component along the axis direction diminishes, while the field component azimuthal to it increases. In a flux rope with cylindrical symmetry, no radial field with respect to the central axis exists. For a special class of flux ropes which can assumed to be force-free, the currents are flowing either in the direction

Published by Copernicus GmbH on behalf of the European Geosciences Union. 

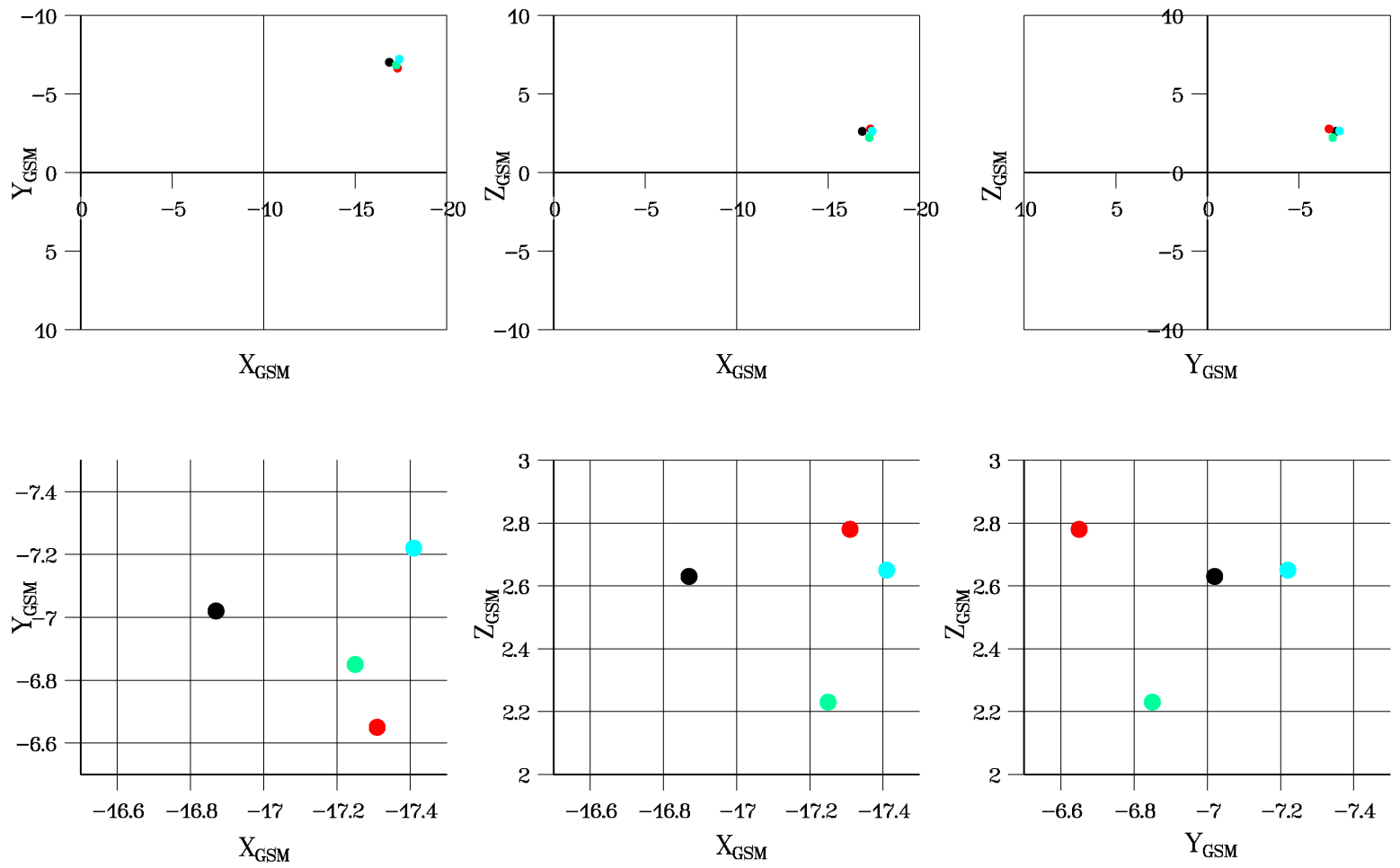

Fig. 1. Cluster spacecraft positions. Upper row: Large-scale view; lower row: Inter-spacecraft separations view. Black: Cluster 1; red: Cluster 2; green: Cluster 3; blue: Cluster 4.

of the magnetic field, or opposite to it. The magnetic field solutions for a force-free, cylindrical flux rope have been given by Lundquist (1950).

Tailward propagating plasmoids and flux ropes associated with them have been observed since the mid-1980's in the magnetotail's plasma sheet using data of the ISEE-3 satellite (e.g. Sibeck et al., 1984) and have later been extensively studied with Geotail data (e.g. Nagai et al., 1998; Ieda et al., 1998; Slavin et al., 1998). In addition to these tailward moving structures, also earthward moving flux ropes have been observed in the near tail $\left(\mathrm{X}>\sim-30 R_{E}\right.$; e.g. Moldwin and Hughes, 1994). Slavin et al. (2003) showed that these flux ropes are often closely associated with earthward bursty bulk flows (BBFs) in the plasma sheet of the magnetotail, i.e., high-speed plasma flows of several $100 \mathrm{~km} \mathrm{~s}^{-1}$ (e.g. Baumjohann et al., 1990; Angelopoulos et al., 1992), and accordingly named them "BBF-type" flux ropes, in contrast to "plasmoid-type" flux ropes moving tailwards. Both types of flux ropes could in many cases reasonably be approximated by a force-free model.

While plasmoid-type flux ropes occur tailward of a major active reconnection $\mathrm{X}$-line and therefore are not magnetically connected to the ionosphere, BBF-type flux ropes occur earthward of such an active reconnection X-line, and are thus at least embedded in an environment which magnetically maps to the ionosphere. The average properties of a BBF-type flux rope with respect to its magnetic field and plasma properties that a satellite encounters during the passage of such a structure have been summarized in a superposed epoch analysis by Slavin et al. (2003): The $\left|\mathrm{B}_{Y}\right|$ component as well as the total magnetic field strength show a peak with a maximum excursion of the order of $\sim 10 \mathrm{nT}$, and a total duration of the excursion of $\sim 30 \mathrm{~s}$. The $\mathrm{B}_{Z} \mathrm{com}-$ ponent simultaneously shows a dipolar structure with a first negative, then positive excursion (this behavior is opposite for plasmoid-type flux ropes). The difference between the opposite $\mathrm{B}_{Z}$ peaks has the same order of magnitude as the $\left|\mathrm{B}_{Y}\right|$ peak. In addition, $\left|\mathrm{B}_{X}\right|$ may also show a unipolar peak like $\left|\mathrm{B}_{Y}\right|$, which is interpreted as a sign of the orientation of the flux rope in the X-Y plane. In the statistical study of Slavin et al. (2003), this azimuthal orientation of a flux rope has been found to be quite variable. Further, an increase of the earthward plasma velocity has on average been observed $\sim 40$ s before the flux rope observation, and this velocity peaks at $\sim 600 \mathrm{~km} \mathrm{~s}^{-1}$ within roughly a minute after the flux rope encounter. Ahead of the flux rope, collocated with the earthward flow increase, a compression region with increased ion densities has been observed. In the lobes neighboring to the flux rope, traveling compression regions (TCR) are formed due to the compression of the lobes by the "obstacle" flux rope and are co-moving with it (e.g Slavin et al., 1984, 2005). 
Flux ropes have been suggested to be part of a "multiple reconnection X-line" (MRX) scenario (Lee, 1995). In this scenario, $\mathrm{N}+1 \mathrm{X}$-lines lead to the formation of $\mathrm{N}$ flux ropes in between them. One of these X-lines is then believed to outpace the others, and will be the first to reconnect open field lines in the lobes. This X-line will then become the major, active $\mathrm{X}$-line in the sense of the "near Earth neutral line" (NENL) substorm model (Baker et al., 1996), and it will divide up the regions of earthward and tailward flows in the magnetotail.

The question what features, if any, may topologically correspond to a BBF-type flux rope in the ionospheric evolution of a substorm has not yet been addressed. This question may in the first view look surprising, as a flux rope is ideally thought to be a closed magnetic structure and thus may not directly map magnetically to the ionosphere. Hence, we would like to stress that we do not claim any features seen in the ionosphere to be directly magnetically connected to the flux rope, but rather inspect whether any topological correspondences exist. Such topological relations may follow from the fact that the flux rope deforms the ambient magnetic field, causes compressions in front of it, inhibits plasma flow through it, etc. E.g., in this respect a TCR is a magnetospheric topological consequence of a flux rope caused by the deformation and compression of the lobe magnetic field lines due to its movement. However, we note that the solutions for a force-free cylindrical flux rope by Lundquist (1950) involve Bessel functions that extend to infinity, and there is no way to "cut" these solutions such that both the conditions of the flux rope being force-free and magnetically closed can be preserved. In the real case, the extension of the flux rope is limited at least by the extension of the magnetosphere. Hence, at least one of the conditions mentioned will not be valid, particularly at the ends of the flux ropes seen along their major axis, which may allow current to flow in or out of the flux rope region.

In order to address the question posed above, in this paper we analyse Cluster satellite data, IMAGE WIC optical data, and conjugate MIRACLE and EISCAT ground-based data for a flux rope event observed by Cluster on 13 August 2002, at $\sim 22: 59$ UT.

\section{Instrumentation and observations}

The Cluster satellite fleet (Escoubet et al., 2001) location and configuration for our event on 13 August 2002, is shown in Fig. 1 for 22:59 UT. Here and in the following, spacecraft 1 is marked with black colour, spacecraft 2 red, spacecraft 3 green, and spacecraft 4 blue. The four spacecraft are situated in the northern near-Earth tail at $\mathrm{X}_{G S M} \sim-17 R_{E}$, in the postmidnight sector (upper row). As can be seen in the lower row of Fig. 1, with $\mathrm{Z}_{G S M} \sim-2.2 R_{E}$ Cluster 3 is situated by $\sim 0.4-$ $0.6 R_{E}$ closer to the equatorial plane than the other three satellites, but takes a central position of the four spacecraft

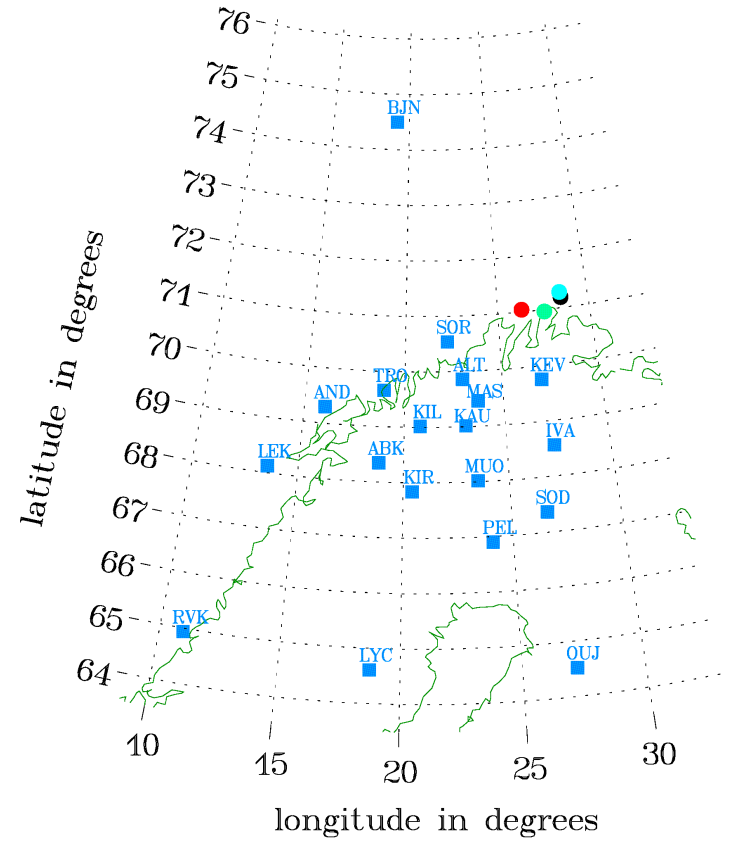

Fig. 2. Mapped positions of Cluster spacecraft to the ionosphere (dots, colours as in Fig. 1), and IMAGE magnetometer stations (squares with station abbreviations).

in the $\mathrm{X}-\mathrm{Y}_{G S M}$ plane. The Cluster footprints in the northern ionosphere together with the ambient IMAGE ground magnetometer network stations (Viljanen and Häkkinen, 1997) are shown in Fig. 2 in the geographic coordinate system. They have been calculated with the Tsyganenko 89 magnetic field model (T89; Tsyganenko, 1989) under the prevailing value of $K p=2$. The validity of the magnetic field modeling using the stated model and parameter has independently been confirmed with the Hybrid Input Algorithm model (Kubyshkina et al., 1999). This model modifies the input to the Tsyganenko model such that it takes into account actual magnetic field measurements from a number of spacecraft in the magnetosphere for our event. The footprints are located above the northern coast of Scandinavia, close to the northeastern edge of the Scandinavian mainland IMAGE stations.

Figure 3a gives an overview over selected Cluster data for the $2 \mathrm{~h}$ surrounding our flux rope event. For our study, mostly data from the Fluxgate magnetometer (FGM, Balogh et al., 2001) and Cluster Ion Spectrometry (CIS, Rème et al., 2001) instruments are used. With respect to the CIS, data from the Hot Ion Analyser (HIA) are shown for spacecraft 1 and 3, while data from the Composition Distribution Function (CODIF) instrument have been used for spacecraft 4 . As can be seen from the increase in $\mathrm{B}_{X}$ (upper panel), the decrease of the ion density (third panel from the bottom) and of the temperature perpendicular to the magnetic field (second panel from bottom), and from the adjustment of the total and 
(a)

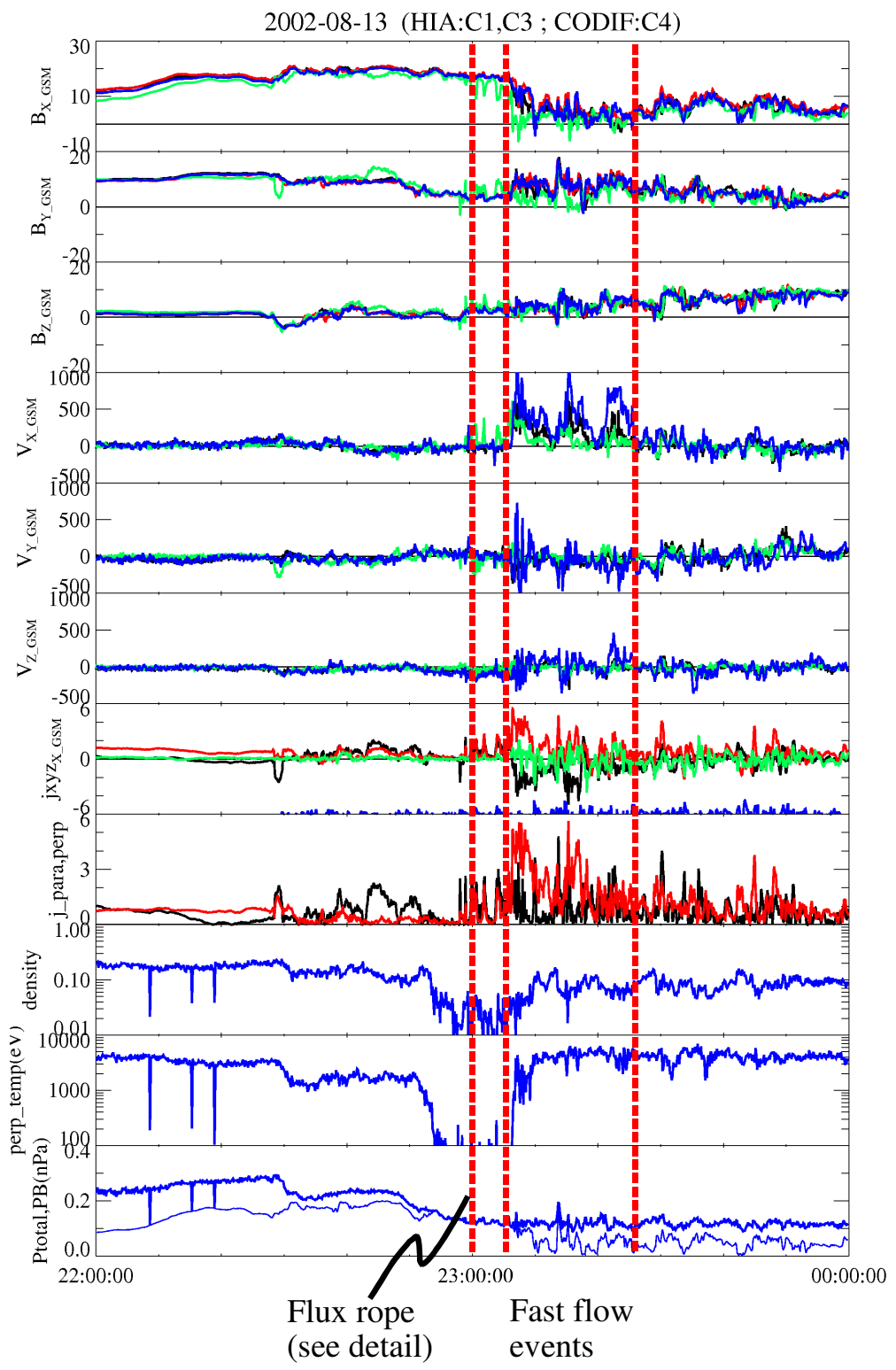

Fig. 3. (a) Cluster FGM and CIS data of 13 August 2002, from 22:00-00:00 UT, for the larger temporal environment of the event discussed. The CODIF instrument has been used for the CIS data of Cluster 4, HIA for CIS data of Cluster 1 and 3; colours for the different Cluster spacecraft as in Fig. 1. Panels from top to bottom: First to third panel: Magnetic field components in nT, in GSM coordinates; Fourth to sixth panel: Ion bulk velocities in $\mathrm{km} \mathrm{s}^{-1}$, in GSM coordinates; Seventh panel: Currents determined with the curlometer technique in $\mathrm{nA} \mathrm{m}^{-2}$, in GSM coordinates: black: X component, red: Y component, green: Z component; magneta: div $\boldsymbol{B}$ (quality parameter, should ideally be zero; plotted with an offset of $-6 \mathrm{nA} \mathrm{m}^{-2}$, for convenience); Eight panel: Currents parallel (black) and perpendicular (red) to the magnetic field, in $\mathrm{nA} \mathrm{m}^{-2}$; Ninth panel: Plasma density at the four different spacecraft, in $\mathrm{ccm}^{-1}$; Tenth panel: Temperature perpendicular to the magnetic field, in eV; Eleventh panel: Total (thick line) and magnetic (thin line) pressure in $\mathrm{nPa}$; the first vertical dashed line marks the flux rope event discussed in more detail in this study, the second and third dashed lines bracket three fast flow events. (b) Similar to (a), but detailed plot for the interval 22:57-23:03 UT, and using 22-Hz magnetic field data; the flux rope event is bracketed by the two vertical dashed lines. (c) Closeup on FGM 22-Hz magnetic field data for a 1-min interval around the flux rope event, in GSM coordinates. (d) Ion velocity distribution functions in three different planes from the CIS/HIA instruments on Cluster 3 at 22:58:51 UT, in GSE coordinates. The logarithmic colour scale represents counts per second. 


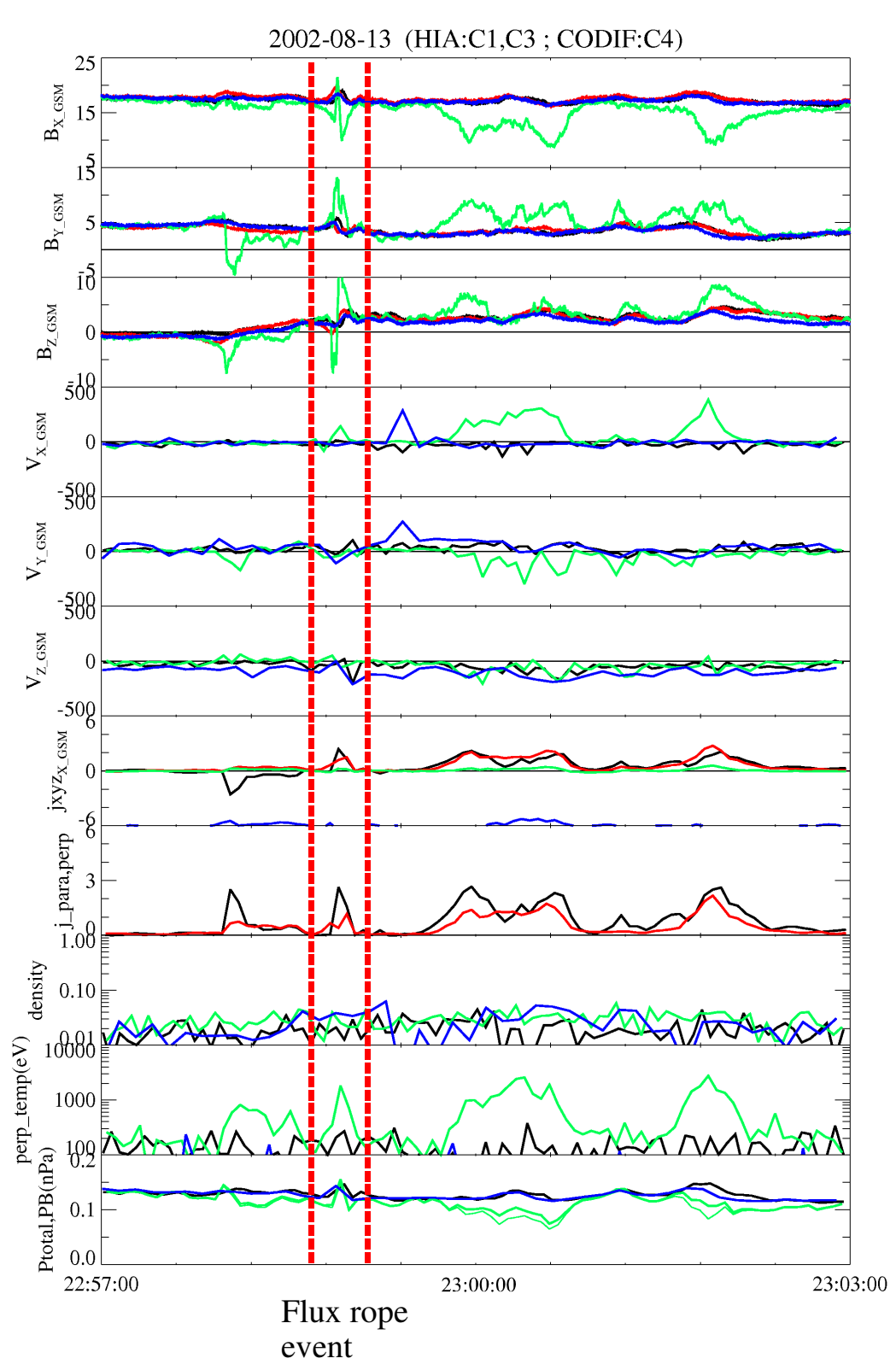

(b)

Fig. 3b. Continued.

magnetic pressure (thick and thin lines in the bottom panel, respectively), a plasma sheet thinning is observed starting from 22:30 UT. During this thinning, all Cluster spacecraft exit to the plasma sheet boundary layer (PSBL). Spacecraft 1, 2, and 4 later exit to the lobe, while spacecraft 3's position undulates between a PSBL-like and a lobe-like region. A weak electron injection onset is seen at one of the LANL geostationary spacecraft at $\sim 22: 50$ UT (data not shown). At $\sim 22: 59 \mathrm{UT}$, the flux rope event which is discussed in more detail below is observed at spacecraft 3 (first vertical dashed line in Fig. 3a). Subsequently, a dipolarization and plasma sheet expansion takes place, and the spacecraft re-enter the plasma sheet, lead by spacecraft 3 at 23:06 UT. During the following $\sim 12 \mathrm{~min}$ after that re-entry, three distinct fast flow events take place with velocities in $\mathrm{X}_{G S M}$ direction up to $\sim 1000 \mathrm{~km} \mathrm{~s}^{-1}$ (see the period between the second and third vertical dashed line in Fig. 3a).

In this paper, we concentrate on the discussion and analysis of the magnetospheric and ionospheric observations in the immediate temporal vicinity of the flux rope event at 22:59 UT. A detailed plot of the Cluster data for a 5-min interval around that event is shown in Fig. 3b, in the same format as Fig. 3a. The flux rope event is bracketed by the two vertical dashed lines. For a detailed discussion of the 


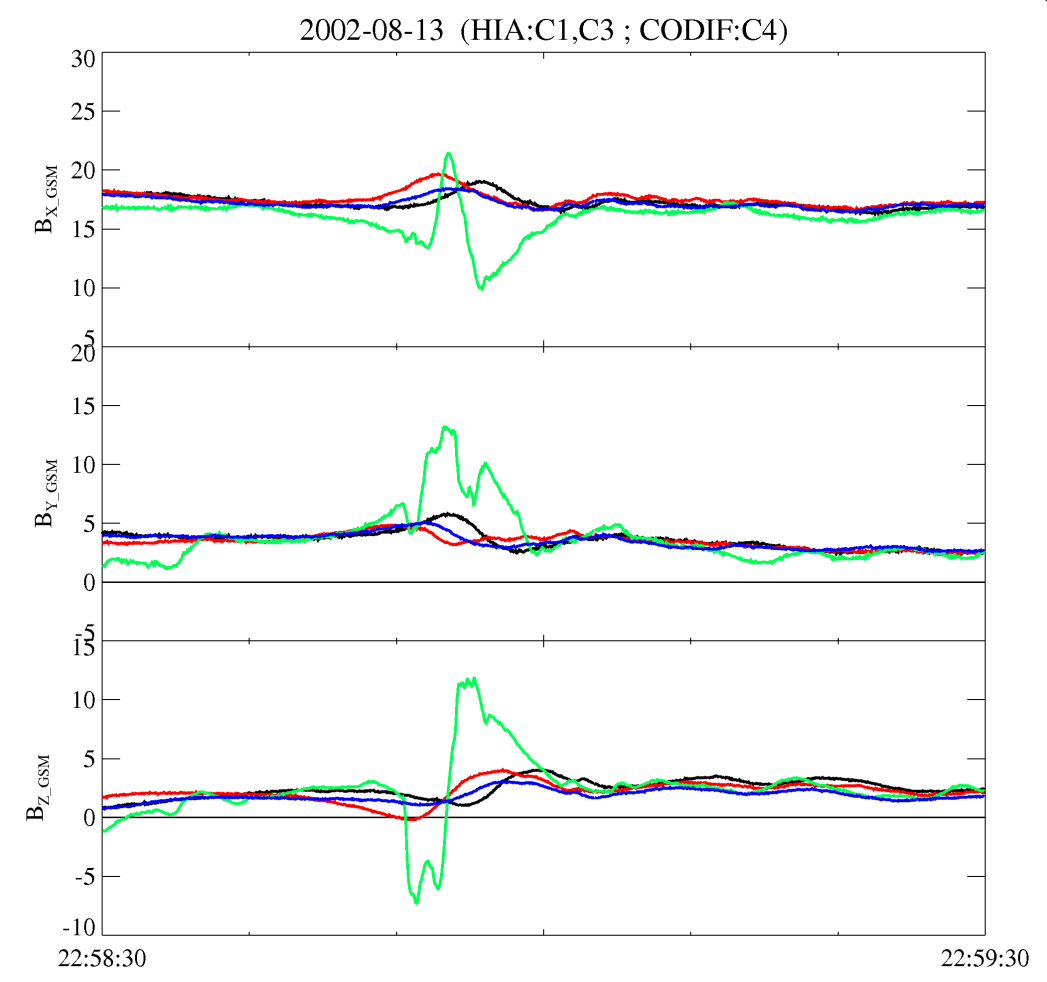

Fig. 3c. Continued.
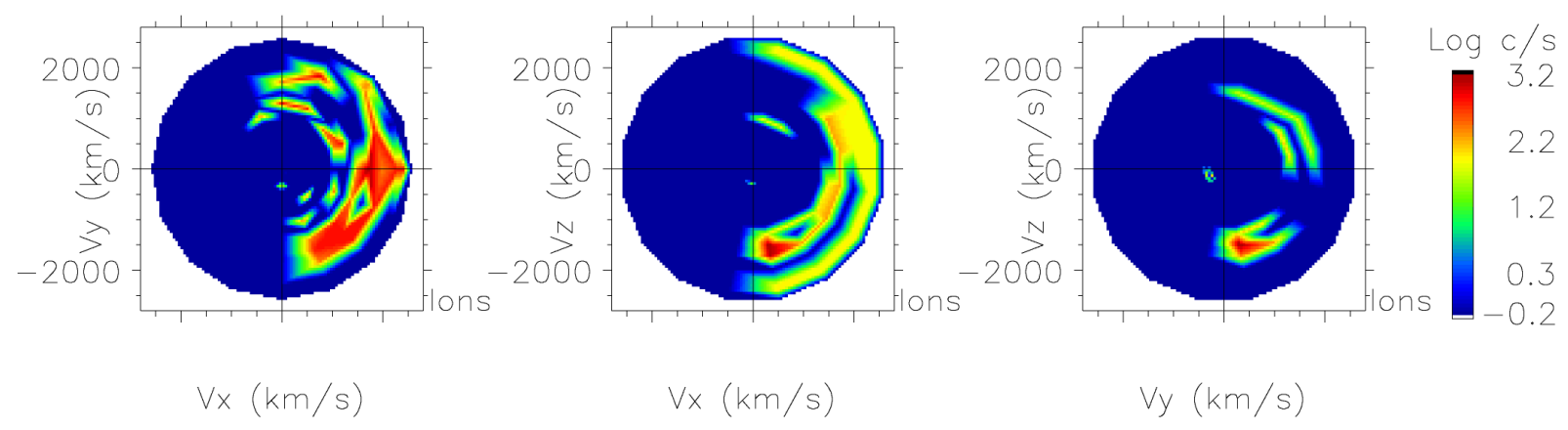

(d)

Fig. 3d. Continued.

magnetic signatures of the flux rope event, the FGM data is shown for a 1 min interval around the event in Fig. 3c. While for Fig. 3a, magnetic data with a time resolution of $4 \mathrm{~s}$ have been used, in Figs. $3 \mathrm{~b}$ and $\mathrm{c}$ the data shown have a $22-\mathrm{Hz}$ resolution. The most pronounced feature in the magnetic data during that period is a positive peak in $\mathrm{B}_{Y}$ of $\sim 9 \mathrm{nT}$ in spacecraft 3, coincident with a bipolar signature of comparable amplitude in $\mathrm{B}_{Z}$. This signature, with a first equatorward, then poleward $\mathrm{B}_{Z}$ component, corresponds to what is expected for a earthward moving or BBF-type flux rope as described in the introduction. Simultaneously with the $\mathrm{B}_{Y}$ increase, a sharp increase of $\mathrm{B}_{X}$ on top of a smoother decrease is measured at spacecraft 3 . While the smooth decrease corresponds to a deeper entry of the spacecraft into the plasma sheet boundary layer, the sharp increase is interpreted as a tilt of the flux rope in the $\mathrm{X}-\mathrm{Y}$ plane. By comparison of the peak values in $\mathrm{B}_{X}$ and $\mathrm{B}_{Y}$ with respect to the background magnetic field, a tilt of $\sim 42^{\circ}$ in clockwise direction (if seen from $+Z$ direction) with respect to the $\mathrm{Y}$ axis is inferred. Also $|\mathrm{B}|$ peaks during the flux rope, as expected (not shown in the figure, but can indirectly be deduced from the peak in the magnetic pressure, bottom panel of Fig. 3b). The duration of the $\mathrm{B}_{Y}$ and $|\mathrm{B}|$ peaks lies with $\sim 20 \mathrm{~s}$ well in the range of the statistical results of Slavin et al. (2003). Simultaneously 


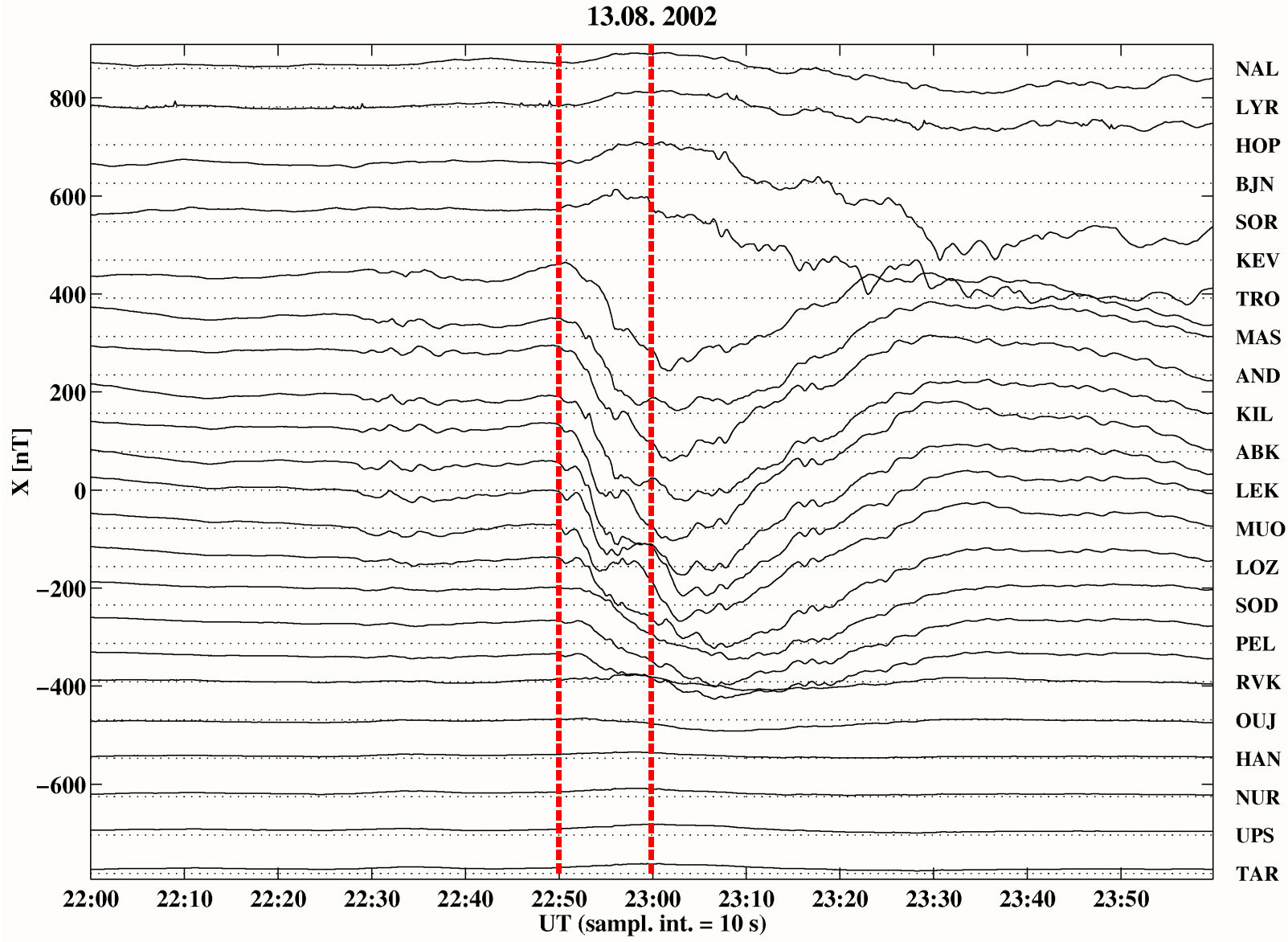

Fig. 4. Geographic north (X) component of IMAGE magnetograms on 13 August 2002, from 22:00-00:00 UT, in nT.

with the flux rope, a downward field-aligned current (FAC) of $\sim 3 \mathrm{nA} \mathrm{m}^{-2}$ is calculated using the curlometer technique (Dunlop et al., 2002; see fourth and fifth panel from the bottom in Fig. 3a).

The other three Cluster spacecraft $(1,2$, and 4$)$ which are located more poleward in the northern lobe do not encounter the FLR, but a corresponding traveling compression region (TCR), as can be seen from the increase in $\mathrm{B}_{X}$ and the dipolar $\mathrm{B}_{Z}$ signature which is smaller, but of similar orientation as the one of the flux rope in spacecraft 3 . From the timing of the $\mathrm{B}_{X}$ peaks, the velocity of this magnetic signature has been inferred to $\sim 1200 \mathrm{~km} \mathrm{~s}^{-1}$ in an earthward and eastward direction in the $\mathrm{X}-\mathrm{Y}$ plane, tilted by $\sim 29^{\circ}$ clockwise from the $+\mathrm{X}$ axis (if seen from $+\mathrm{Z}$ direction). This value is well in accordance with the results of the TCR statistics by Slavin et al. (2005). As the direction of the core of the flux rope is expected to be roughly $90^{\circ}$ tilted with respect to the motion of the associated TCR, this leads to an estimate for the flux rope tilt of $\sim 29^{\circ}$ clockwise from the $+\mathrm{Y}$ axis (if seen from $+\mathrm{Z}$ direction), which is somewhat smaller than the value of $42^{\circ}$ deduced from the Cluster 3 data above. However, since Cluster 3 is located northward of the core of the flux rope, for the given geometry this increase of the apparent tilt angle with increasing distance from the core in $+\mathrm{Z}$ direction is expected.

While only a slight increase of the earthward flow velocity up to $\sim 100 \mathrm{~km} \mathrm{~s}^{-1}$ is seen in the ion moments of Cluster 3 in association with the flux rope event (Fig. 3b), the ion velocity distribution functions derived from the CIS/HIA instrument on Cluster 3 at 22:58:51 UT (Fig. 3d) reveal a clear highspeed flow, exceeding $1500 \mathrm{~km} \mathrm{~s}^{-1}$, with a mainly earthward $\left(\mathrm{V}_{X}\right)$ and some eastward $\left(\mathrm{V}_{Y}\right)$ component. This direction of the highspeed flow agrees well with what is expected from the timing of $\mathrm{B}_{X}$ as discussed above. Yet, this flow is mixed with a mainly equatorward $\left(-\mathrm{V}_{Z}\right)$ flow that has smaller $\mathrm{V}_{X}$ and $\mathrm{V}_{Y}$ components. Due to this multicomponent feature in a highly fluctuating field the moments shown in Fig. $3 \mathrm{~b}$ do not represent the flux-rope associated fast flow.

The X (geographic north) component of the IMAGE magnetometers are shown in Fig. 4 in a conventional magnetogram plot. Following a rather moderately disturbed period, the pre-existing westward electrojet intensifies at 22:50 UT in all northern Scandinavian mainland stations (simultaneously with the observed electron injection at LANL; see first 


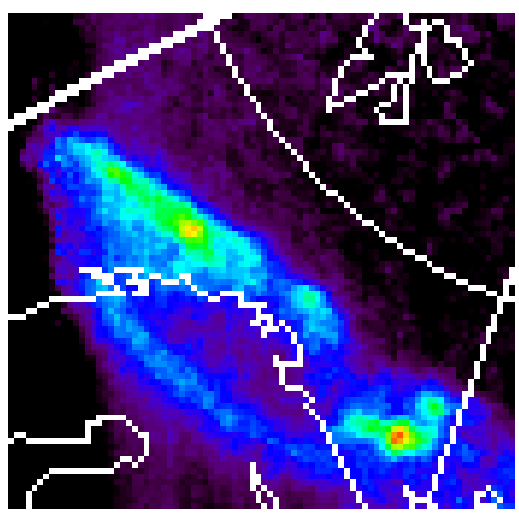

2256:38 UT

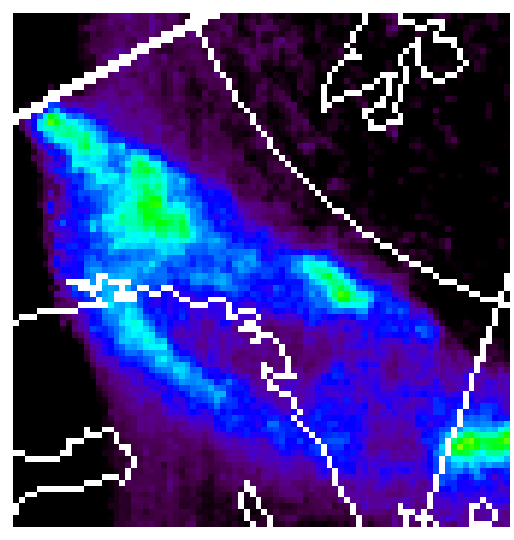

2258:41 UT

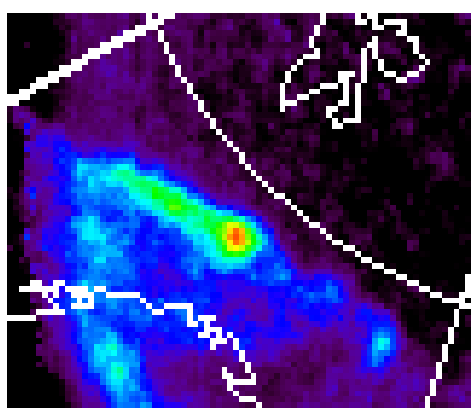

2300:43 UT

Fig. 5. Detailed view of the FUV emissions over the Scandinavian area from the WIC camera on the IMAGE satellite, for three consecutive timesteps around the flux rope event.
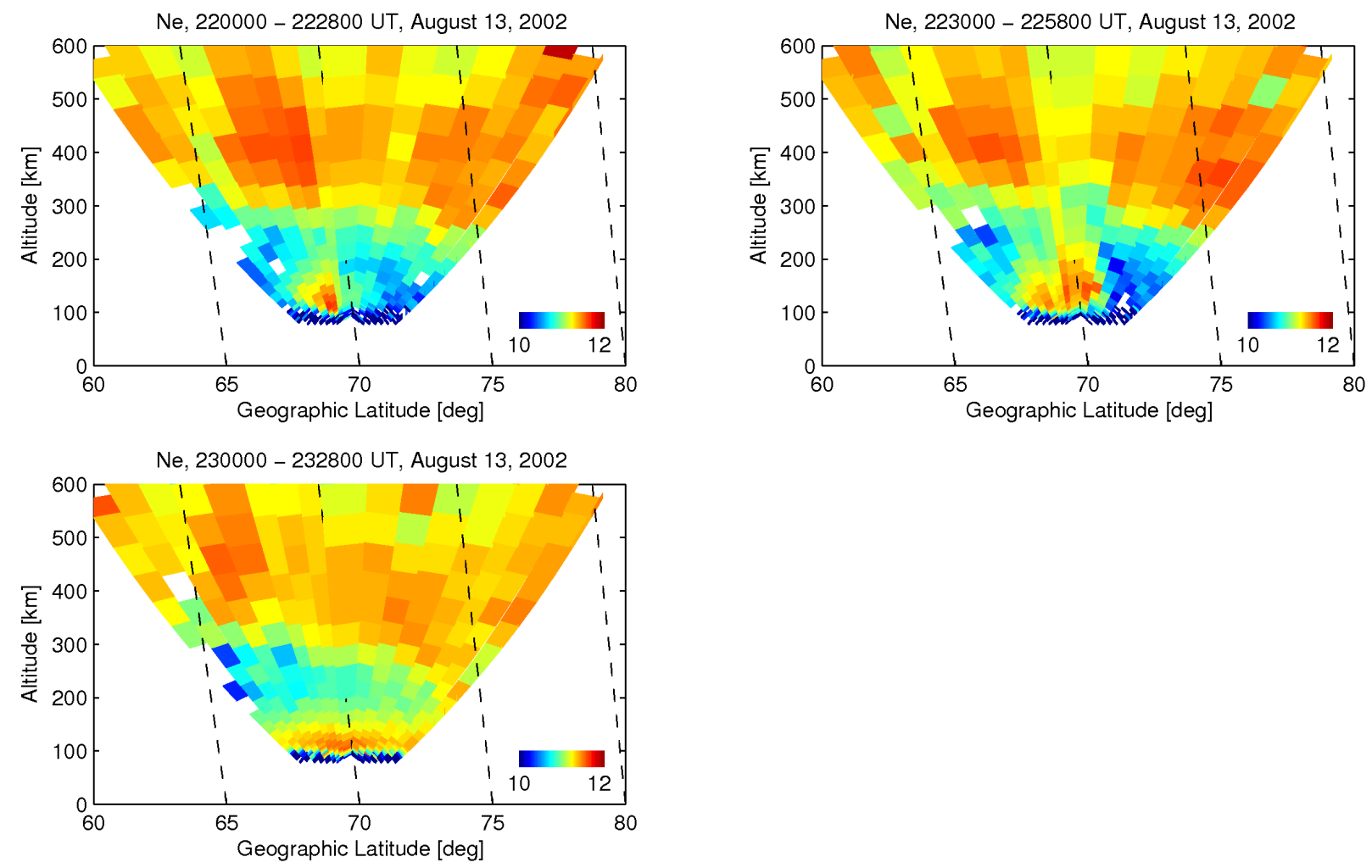

Fig. 6. EISCAT electron density data in $\log \left(\mathrm{m}^{-3}\right)$, as a function of geograhic latitude and altitude, for three consecutive scans from north to south (for the longitudes of the beam at $114 \mathrm{~km}$, see Fig. 10).

vertical dashed line in Fig. 4). The flux rope observation at Cluster occurs during the late expansion phase of the substorm as observed in IMAGE (see second vertical dashed line in Fig. 4). Note that some of the stations in the northeastern Scandinavian mainland, such as Masi (MAS) and Kevo (KEV), show a clear positive excursion which peaks at $\sim 23: 00$ UT. Subsequently, the recovery phase begins which lasts until $\sim 23: 25$ UT. The ground magnetic data will be analysed in more detail in the following section.

The Far Ultraviolet Wideband Imaging Camera (WIC) on the IMAGE satellite monitors the auroral emissions in the spectral range of 140-190 nm (Mende et al., 2000). On 

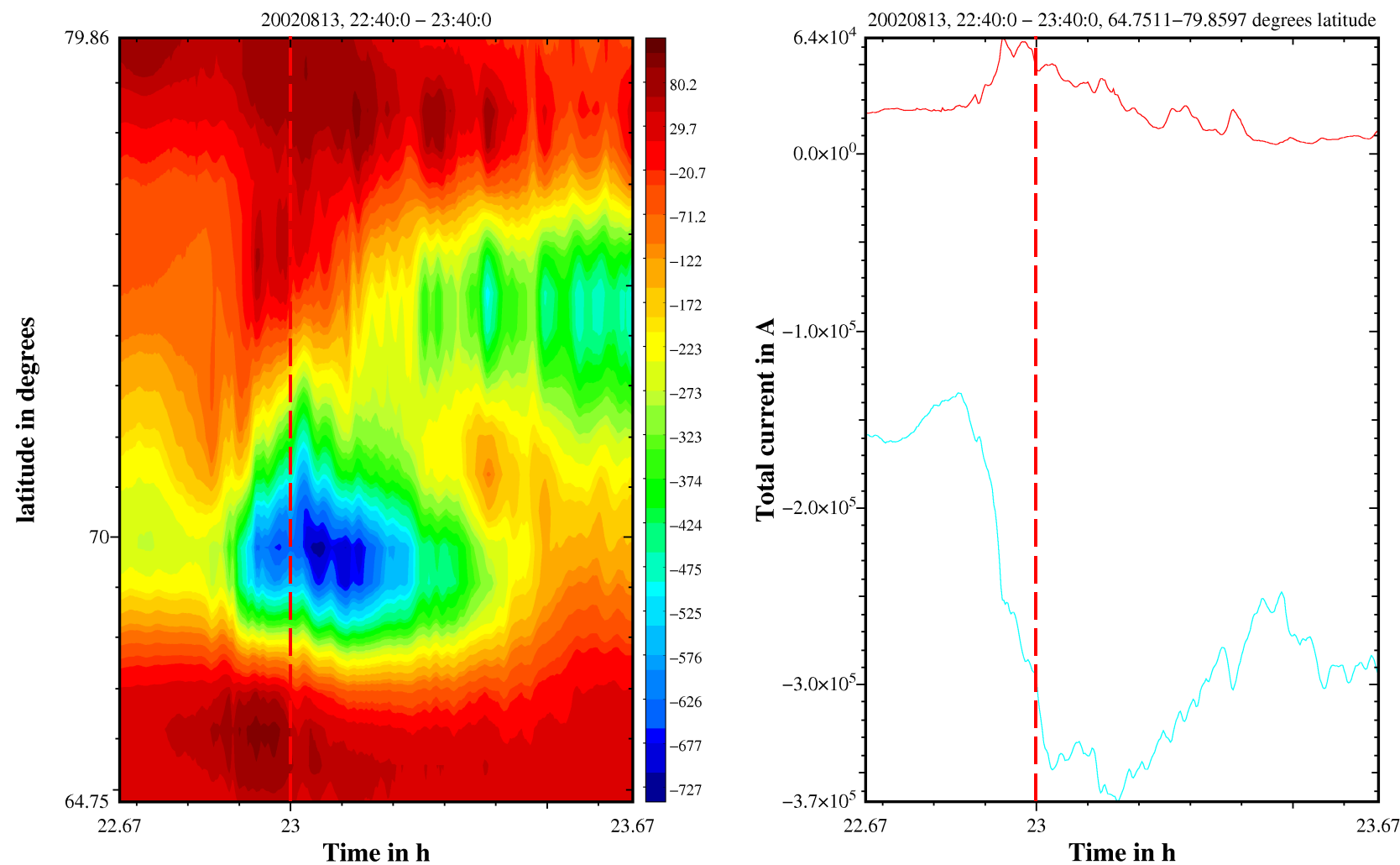

Fig. 7. Left panel: Ionospheric equivalent currents, as derived by the 1-D SECS upward continuation technique from the ground magnetic data, in $\mathrm{mA} \mathrm{m}^{-1}$, from 22:40-23:40 UT. The dashed red line marks the time of the flux transfer event. Right panel: Total integrated westward and eastward currents as a function of time

13 August 2002, the field-of-view of the WIC covered almost the whole northern auroral region, with Northern Scandinavia located close to the limb of the observation area, and this limb shifting polewards during the time of interest around 23:00 UT. Therefore the format of the individual WIC images of Northern Scandinavia shown in Fig. 5 changes slightly, but the area of main interest of our study, i.e. the region around the northern Scandinavian coast, is visible throughout. A geographic coordinate system and coastlines have been superposed to the images. Two major auroral regions are visible: an equatorward, fainter, auroral structure is seen located at $\sim 68^{\circ}$ of latitude. This structure has been pre-existing before the substorm intensification at 22:50 UT (data not shown). Starting from that substorm onset, a second, brighter auroral region has developed and moved polewards. At 22:56:38 UT, this regions peaks at $\sim 71.2^{\circ}$ latitude, while at 23:00:43 UT, it has reached $\sim 72.7^{\circ}$ latitude at $16^{\circ}$ longitude. To the west and to the east of Scandinavia, these two auroral regions appear to re-unite, forming a convex lense-like shape. In the center of this lense, an area with clearly less auroral emission is observed, which at 22:58:41 UT is located just at the northern coastline of Scandinavia. At 23:00:43 UT, this area is again covered with moderate auroral emission, while the "void" area may have traveled eastwards (however, this cannot be unambigously concluded because of the border of the WIC field-of-view).

The European Incoherent Scatter Facility mainland radar (EISCAT; Folkestad et al., 1983) was operating in a meridional scanning mode during our event such that the main radar beam is moved equatorwards from $72.2^{\circ}$ latitude to $67^{\circ}$ latitude (at $117-\mathrm{km}$ altitude) during $28 \mathrm{~min}$, and then the antenna is directed back to the starting position within the following $2 \mathrm{~min}$, in order to complete a full 30-min cycle. During our period of main interest, these cycles started at 22:30 and 23:00 UT, respectively. The main beam data are used to deduce, among others, the electron density, ion and electron temperatures, and ion velocity in the beam direction. By using all three EISCAT sites including the receiving stations at Kiruna and Sodankyla, also the full ion velocity vector in the F-region (at 293-km altitude) and thus the electric field was derived. Figure 6 shows the electron density distribution as a function of geographic latitude and altitude for three subsequent cycles. The direction of geomagnetic field lines is indicated by black dashed lines. The higher altitude $(>250 \mathrm{~km})$ ionisation caused by soft precipitating particles and remnant ionisation is comparably uniform and of less interest here. The scan 22:00-22:30 UT (upper left panel) represents the background conditions, during which enhanced low-altitude 


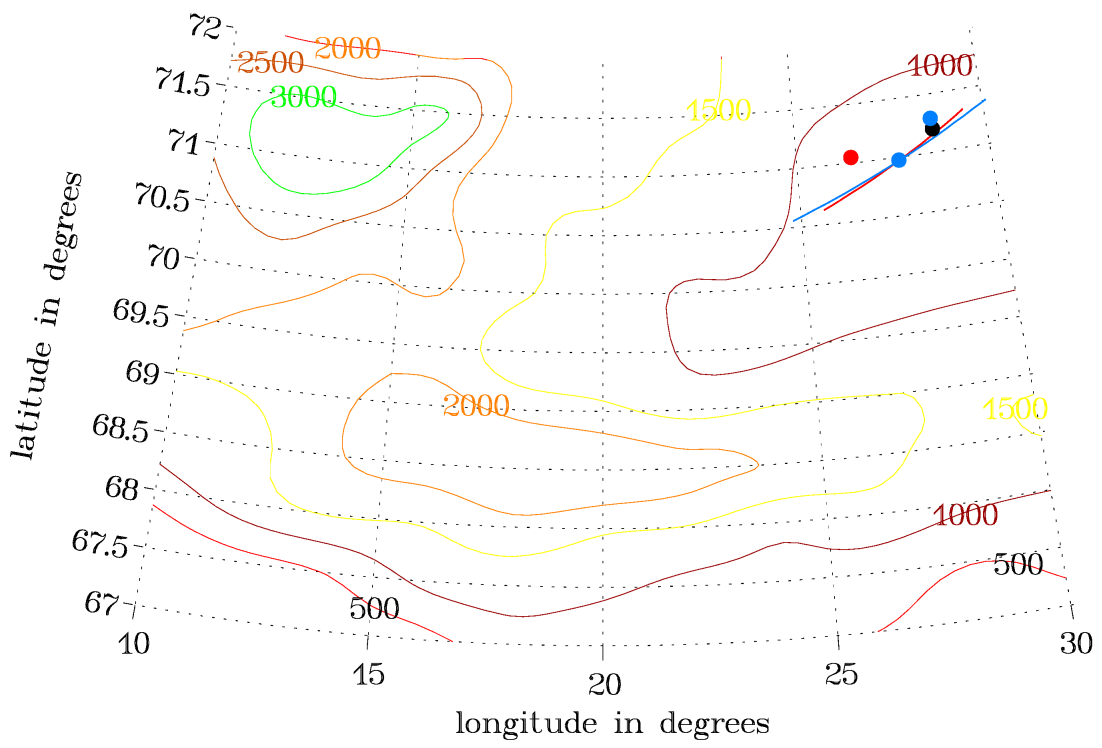

Fig. 8. Cluster footprints (dots, colours as in Fig. 1), and direction estimates of the flux rope as mapped to the ionosphere (blue line: based on $90^{\circ}$ rotated TCR direction of motion; red line: based on the orientation measured by Cluster 3; in both cases linearly continued in the magnetospheric X-Y plane) at 22:59:50 UT, together with WIC emissions in R at 22:58:41 UT (iso contour lines).

ionisation was only present around $68^{\circ}$ latitude, i.e., in the area of the equatorward auroral structure seen by WIC. During the second cycle (upper right panel), the substorm intensification initiates when the beam is located at $\sim 68.2^{\circ}$ latitude. The ionisation poleward of that, reaching to $\sim 71^{\circ}$ latitude, is thus recorded before that intensification, and shows that already in the growth phase energetic particle precipitation was present poleward of the equatorward auroral structure. During the third cycle (lower right panel), i.e., after the intensification, low-altitude ionisation is observed over the whole latitude range measured by the radar. The heightintegrated ionospheric conductances derived from these data as well as the electric field structure will be discussed in the next section.

\section{Analysis results and discussion}

In order to obtain a clearer picture about the evolution of the ionospheric currents during the substorm in which the flux rope event occurred, Fig. 7 shows the ionospheric equivalent currents in east-west direction, upward continued from the ground magnetic measurements along a north-south aligned chain of magnetometers with the 1D Spherical Elementary Current System (SECS) technique (Vanhamäki et al., 2003). This technique derives the ionospheric equivalent current in a spherical geometry as a funtion of latitude, assuming that derivatives in longitudinal direction vanish. In the left panel of Fig. 7, the eastward equivalent currents are shown in red colour, while westward equivalent currents are displayed in orange to blue colours. Clearly seen is the activation at
22:50 UT and the following broadening and intensification of the westward electrojet. Interestingly, the location of the strongest current flow does not significantly shift poleward, but rather remains at $\sim 69.5^{\circ}$ latitude. However, the width of the area of strong westward current significantly increases, and its northward boundary is located at $\sim 72.8^{\circ}$ at 23:00 UT, in good agreement with the expansion of the poleward auroral structure seen by WIC. After 23:03 UT, the width of the westward electrojet region again decreases in the recovery phase, before another activation sets on further polewards at $\sim 23: 15$ UT. If we inspect the moment of the flux rope encounter at Cluster shortly before 23:00 UT (dashed vertical line in Fig. 7), no specific signature of the flux rope event is seen in this $1 \mathrm{D}$ view. Similarly, the total integrated westward and eastward currents along the 1D chain (right panel in Fig. 7) does not show any specific inhibition (nor increase) of the total current due to the flux rope event. At 23:00 UT, the substorm is in its late expansion phase, and the integrated westward current density (blue line) continues to increase until it reaches its absolute maximum of $370 \mathrm{kA}$ at $\sim 23: 10 \mathrm{UT}$. Hence we conclude that in a 1-D and integrated representation, no specific counterpart of the flux rope event is seen in the ionospheric currents.

The picture changes when we inspect the 2-D distributions of the ionospheric quantities: Fig. 8 shows the WIC intensities over Northern Scandinavia together with the mapped location and direction of the flux rope, as well as the Cluster magnetic footprints. As before, the mapping has been carried out with the Hybrid Input Algorithm model. Although during our disturbed substorm interval it is not easy to apply 

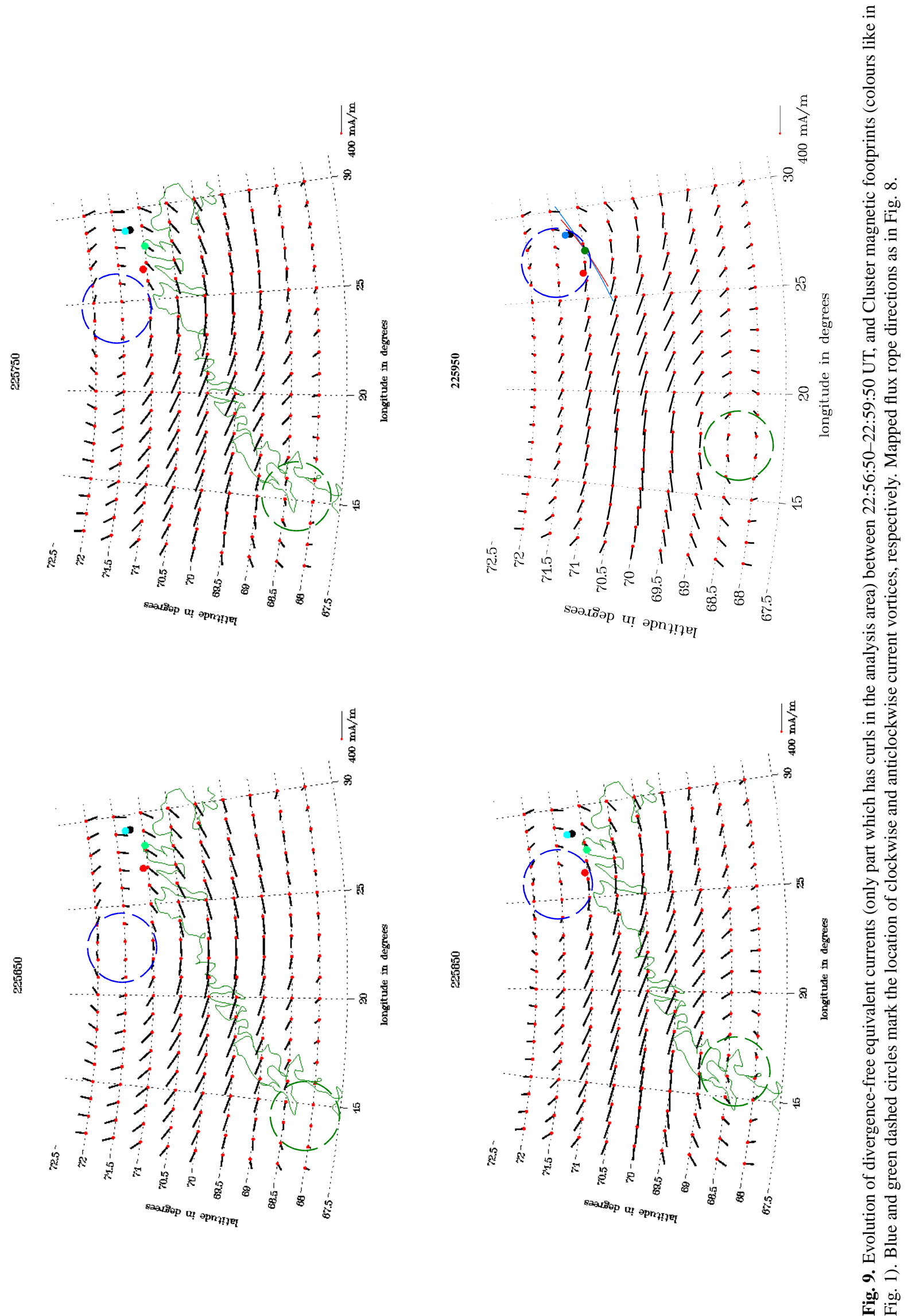
morphological mapping arguments of the auroral structures, we tentatively interprete the morphological structure of the aurora as follows: The poleward boundary where the moderately bright aurora $(\sim 1500 \mathrm{R})$ is roughly uniform in longitude is located at between $69^{\circ}$ and $69.5^{\circ}$ latitude (Fig. 8). This aurora may be interpreted as stemming from plasma sheet precipitation earthward of the FLR. Cluster 3 is located poleward of this main plasma sheet region in a region of little auroral precipitation, as is supported by the low $\beta$ value it observes during the FLR event (see the observed absolute and magnetic pressure, Fig. 3b). Thus our magnetic mapping results are well consistent with morphological arguments. Note that for any features at and tailward of the FLR, the FLR obstacle will perturb the morphology of the particle precipitation.

For the flux rope mapping, the blue line in Fig. 8 shows the estimate from the $90^{\circ}$ rotated direction of the TCR motion, and the red line the estimate as determined from the Cluster 3 magnetic data. In both cases, for the mapping the estimated directions were linearly continued in the magnetospheric X$\mathrm{Y}$ plane. The flux rope maps into the lens-shaped area of weak FUV emissions below 1000 R (cf. also Fig. 5). Moreover, also the orientation of the mapped flux rope coincides well with that of the weak auroral emission region, which are both northeast-southwest aligned in the ionosphere. Note that although the $90^{\circ}$ rotated direction of the TCR motion is expected to correspond best to the flux rope core direction, this conclusion is qualitatively not sensitive to the difference between the two estimates of the flux rope orientation. Since a flux rope effectively acts as an "obstacle" for particles precipitating from the tail to the ionosphere, it is likely that this signature is caused by an inhibition of such particles due to the presence of the flux rope.

A further topological correspondence to the flux rope is found in the 2D ionospheric current pattern. For that purpose, we calculate the 2-D ionospheric equivalent currents $\boldsymbol{J}_{\text {eq,ion }}$ using the 2-D SECS technique for the upward continuation of the ground magnetic field (Amm and Viljanen, 1999). Further, we decompose this currents according to $\boldsymbol{J}_{e q, i o n}=\boldsymbol{J}_{d f, i o n}+\boldsymbol{J}_{\text {lap,ion }}$, where $\boldsymbol{J}_{\text {lap,ion }}$ is the part of the current which is divergence- and curl-free within our analysis area, while $\boldsymbol{J}_{d f, i o n}$ denotes the part of the current which is divergence-free, but not curl-free inside that area (e.g. Amm, 1997). (Note that $\boldsymbol{J}_{e q, i o n}$ is by definition divergence-free, cf., e.g., Untiedt and Baumjohann, 1993.) With such a decomposition, the main background westward electrojet will be contained in $\boldsymbol{J}_{\text {lap,ion, }}$, while local features of the current system are seen in $\boldsymbol{J}_{d f \text {,ion. }}$. The evolution of this latter current system is shown in 1-min steps in Fig. 9, between 22:56:50 and 22:59:50 UT. Two oppositely directed current vortices are visible, a clockwise one which is centered at $\sim 71.5^{\circ}$ latitude, and an anticlockwise one which is centered slightly poleward of $68^{\circ}$ latitude. If the ionospheric conductances were uniform, the clockwise vortex would correspond to a downward FAC, and the anticlockwise one to an upward
FAC. Both vortices are moving synchronously eastward, with a speed of $\sim 2 \mathrm{~km} \mathrm{~s}^{-1}$. The poleward vortex is reaching the Cluster satellites just at the time when a downward FAC is also observed in the Cluster data (cf lower right panel in Figs. 9 and 3b). Comparing the location of the two vortices at 22:58:50 UT with the FUV image which was taken $10 \mathrm{~s}$ earlier (Fig. 8), it can be seen that the anticlockwise vortex is well collocated with the lens-shaped emission minimum, while the clockwise vortex is well collocated with the emission maximum around $68.5^{\circ}$ latitiude, which supports the interpretation of the current vortices as downward and upward FAC regions, respectively. We notice that the tilt of the axis between the two current vortices is comparable with the tilt of the ionospheric projection of the flux rope direction (the two estimates are marked by the blue and red lines in the 22:59:50 UT panel of Fig. 9, similarly to Fig. 8). Furthermore, with $\sim 2.4 \mathrm{~km} \mathrm{~s}^{-1}$, the mapped eastward bulk velocity observed at Cluster 4 (which is located closest to the central latitude of the anticlockwise vortex) agrees well with the eastward speed of the current vortices.

It may be noted that during the latter fast flow events starting at $\sim 23: 00$ UT, downward FAC are also observed by Cluster (Fig. 3b). The FAC at the FLR event is, however, mostly caused by a very localised change in the $\mathrm{dB}_{Z} / \mathrm{dY}$ component, and thus leads to a localized FAC pattern in the ionosphere, while the FAC during the latter fast flow events mostly stem from $\mathrm{dB}_{Y} / \mathrm{dZ}$ which suggests dawn-dusk extended current sheets.

Thus we conclude that in a 2-D view, there are several clear topological ionospheric correspondences to the flux rope event: A lens-shaped decrease of the ionospheric FUV emissions which is collocated with a downward FAC area that moves eastward with a speed close to the bulk velocity of the plasma as measured in the magnetosphere. This leading downward FAC area is balanced by a trailing upward FAC area which moves eastward at the same speed at lower latitudes. On the other hand, no ionospheric topological correspondence is found to the mentioned fast earthward motion of the $\mathrm{B}_{X}$ peaks of the TCR, which would map to a $\sim 22 \mathrm{~km} \mathrm{~s}^{-1}$ eastward velocity component in the ionosphere.

A tentative interpretation of the topological correspondence might be that the two current vortices in $\boldsymbol{J}_{d f, i o n}$ are the "edges" of a flux rope in its symmetry direction where part of the current flowing in its central area is diverted into upward and downward FAC, respectively. Both in the ionosphere and in the flux rope (where $\boldsymbol{j} \| \boldsymbol{B}$ ), the currents are flowing southwestwards. (Note that the $\boldsymbol{J}_{d f \text {,ion }}$ currents in Fig. 9 would in case of uniform conductances correspond to Hall currents, but the total currents related to the double vortex structure are expected to flow from the downward FAC area in the northeast to the upward FAC area in the southwest. This is also discussed below when looking at the ionospheric electric field.) For the flux rope, this current direction also corresponds to the prevailing positive IMF $\mathrm{B}_{Y}$ component (data not shown). Hence, in this interpretation there is 


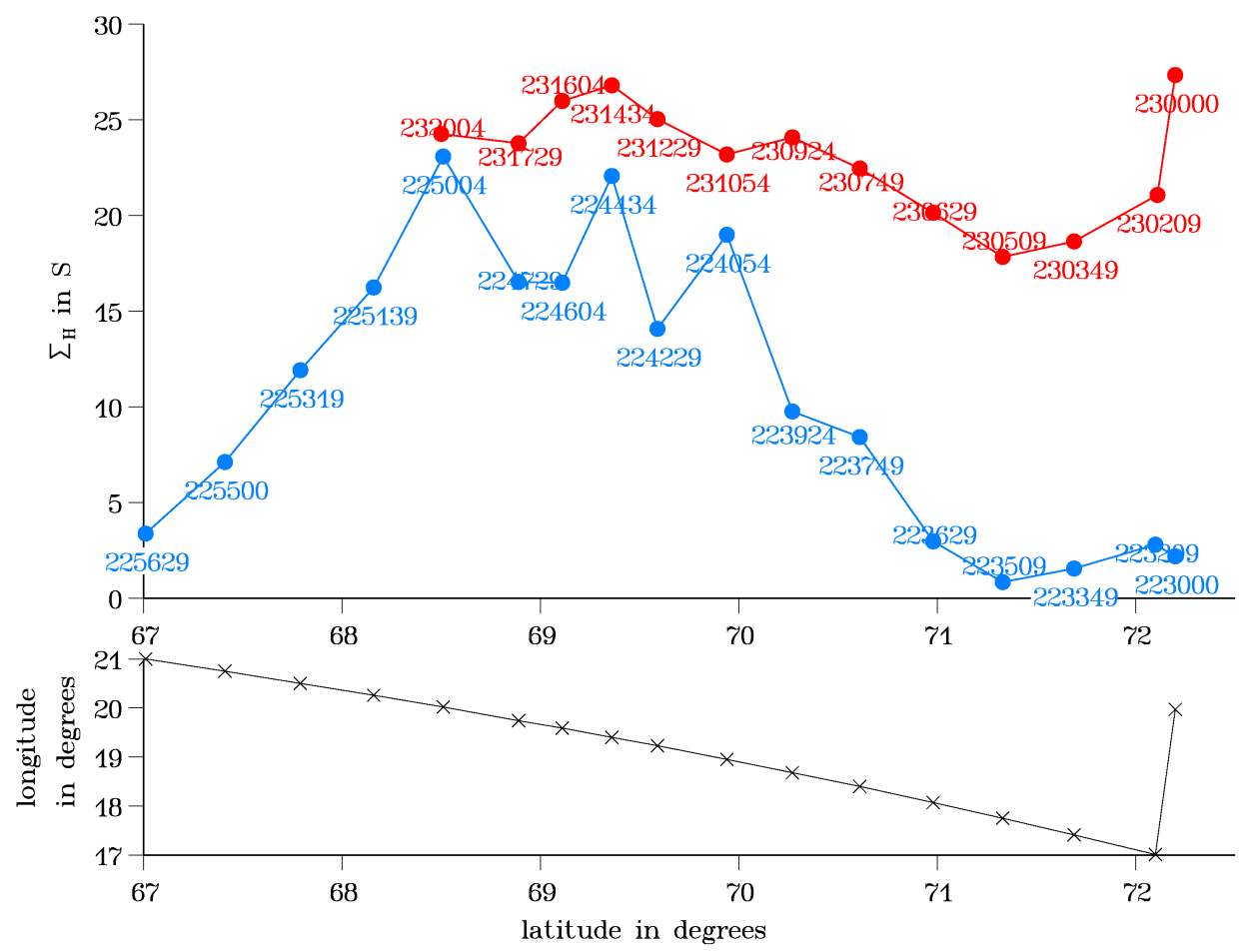

Fig. 10. Upper panel: EISCAT height-integrated Hall conductance values (integrated along the radar beam, which is not necessarily fieldaligned), in S, for two consecutive scans from north to south. Blue: Scan mostly before substorm activation at 22:50 UT; red: Scan after substrom activation. Numbers at the dots (measurement points) mark the times of the observations. Lower panel: Latitude and longitude of the radar beam at $114 \mathrm{~km}$ for each measurement point.

no current circuit between ends of the flux rope and the ionosphere, since in this case the current direction in both domains should be opposite, and such a circuit would miss a current generator. Rather, the current generated further tailward in the magnetosphere could close either via the ionosphere, or close by "tunneling" through the flux rope, in the same direction. If this interpretation is correct, the role of the flux rope in this picture should depend on the $\mathrm{B}_{Y}$ component prevailing in it, since only for a positive $\mathrm{B}_{Y}$ the currents could be closed in the direction as required during a substorm. We emphasize that this interpretation is at the present stage speculative, and more work needs to be done to either verify or falsify it. However, it would be consistent with the topology of the horizontal currents and FAC, as observed by Cluster and in the ionosphere.

Finally, we return to a 1-D view of the ionosphere and inspect two important parameters which cannot be retrieved from the MIRACLE data for our event, since only one of the two STARE radar stations was operative: the ionospheric conductances and the electric field as obtained from EISCAT data. The upper panel of Fig. 10 shows the "beamaligned" Hall conductance $\Sigma_{H, \text { beam }}$ which has been integrated along the respective EISCAT beam direction, and thus is not necessarily field-aligned. The numbers at the observation points indicate the observation time of each point, and the geographic longitude of the observations is plotted in the lower panel. The blue curve in the upper panel shows the $\Sigma_{H, \text { beam }}$ scan which was mostly taken before the activation at 22:50 UT. It shows larger Hall conductances over $15 \mathrm{~S}$ only in the geographic latitude range of $\sim 68-70^{\circ}$, and very low Hall conductances less than $5 \mathrm{~S}$ poleward of $71^{\circ}$ latitude. After the activation, $\Sigma_{H, \text { beam }}$ has increased to values larger than $20 \mathrm{~S}$ almost along the whole latitude range of $68.5-72.2^{\circ}$. However, a minimum of $\Sigma_{H, \text { beam }}$ is encountered at $71.3^{\circ}$ lat, which is the same latitude where about $5 \mathrm{~min}$ before the lensshaped FUV emission minimum region and the clockwise $\boldsymbol{J}_{d f \text {,ion }}$ vortex associated with downward FAC has passed. Even though these EISCAT observations were made $\sim 5 \mathrm{~min}$ after the center of the FUV emission minimum has passed, and somewhat westwards of the location of that minimum during the flux rope event, we interpret the existence of the conductance minimum as a confirmation that, compared to the environment, lower ionospheric conductances prevail in the area of the FUV emission minimum and downward FAC loop, as to be expected.

The electric field observations by EISCAT are shown in a superposed vector plot in Fig. 11. The measurements of the 22:30-23:00 UT scan are shown at their actual observation points, while measurements of later scans have been shifted eastward in space, and those of earlier scans 


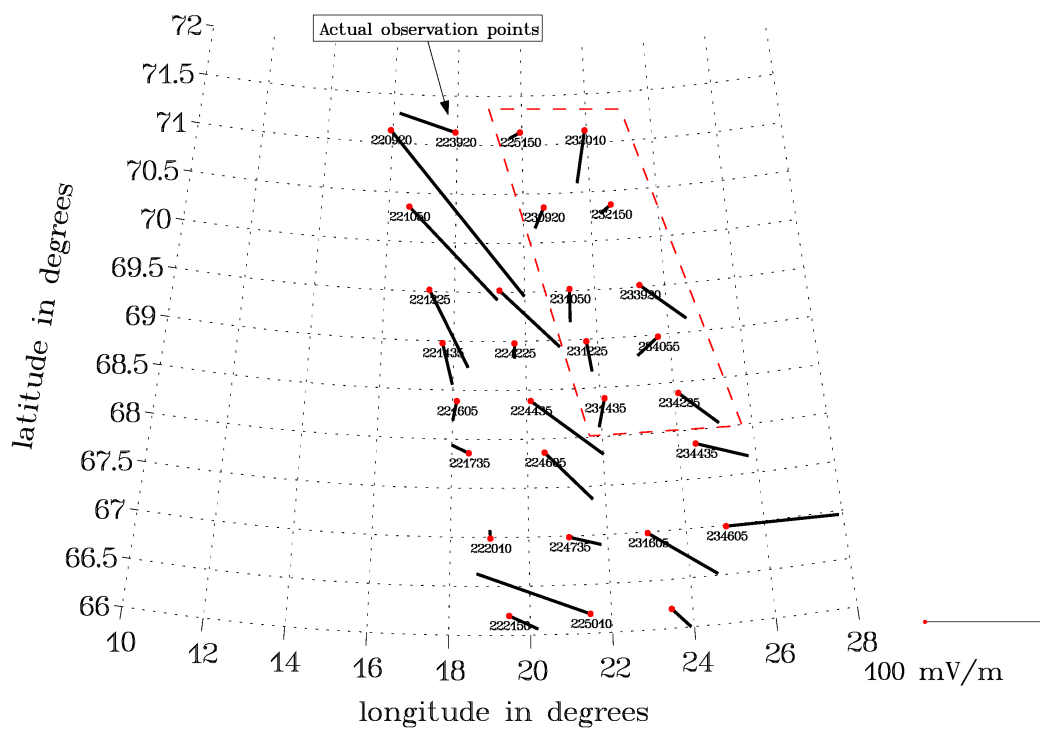

Fig. 11. Electric field measured by EISCAT for four consecutive scans from north to south between 22:00-00:00 UT (two vectors have been deleted for data quality reasons). Data of the scan 22:30-23:00 UT are shown at the actual observation points, data of earlier scans are shifted westward, of later scans eastward. The area enclosed by the red dashed line marks the spatio-temporal domain of the substorm intensification and recovery phase.

westwards. Again, the observation times are indicated at the vectors. We note that before and later equatorward of the substorm region, large southeastward pointing electric field vectors with magnitudes partly well above $100 \mathrm{mV} \mathrm{m}^{-1}$ are seen. This high electric field region, which is independently also confirmed by a comoving area of enhanced ion tempe- ratures (data not shown), moves equatorward with a speed of $\sim 0.1 \mathrm{~km} \mathrm{~s}^{-1}$. However, as this interesting feature is pre-existing to the substorm, its detailed analysis lies outside the scope of this paper. We concentrate on the electric field structure after the activation and inside the substorm area (i.e., poleward of $\sim 68^{\circ}$ latitude) which is marked by the red box in Fig. 11. Especially during the 23:0023:30 UT scan, the electric field magnitudes are depressed to values of $20-30 \mathrm{mV} \mathrm{m}^{-1}$, as to be expected due to the conductance enhancement during the substorm. During this first scan, the electric field points mostly southward, which is just the electric field direction required to direct the total horizontal ionospheric current into a west-southwestward direction, in good correspondence with the direction between the downward and upward FAC regions in Fig. 9. During the 23:30-00:00 UT scan, i.e., already at the end and after the recovery phase of the substorm (cf. Fig. 7), the electric field magnitudes somewhat increase again and the directions become more variable. Hence we conclude that the EISCAT electric field data during the substorm event is compatible with the current structure found from the magnetic field data, and thus further confirms our interpretation of the two current vortices as oppositely directed FAC regions.

\section{Summary and conclusions}

During the expansion phase of a substorm on 13 August 2002, around 22:59:50 UT Cluster 3 observed an earthward traveling BBF-type flux rope in the PSBL, while the other three Cluster spacecraft experienced the signature of a TCR in the northern lobe. Our search for possible topological correspondences in the conjugate ionosphere to these magnetospheric substorm features has yielded the following results:

- At the ionospheric footprint of the flux rope, a convex lens-shaped FUV emission minimum region is observed inside which the ionospheric conductances are diminished with respect to the environment. The alignment of the lens shape corresponds well to the mapped direction of the flux rope.

- This FUV emission minimum region is collocated with a downward FAC area as both measured with Cluster and inferred from the ground-based data. The downward FAC area is moving eastward with a speed of $\sim 2 \mathrm{~km} \mathrm{~s}^{-1}$ which is in good agreement with the mapped bulk velocity measured at the Cluster spacecraft closest to that area.

- Comoving with this leading downward FAC area at $\sim 71.5^{\circ}$ latitude is a trailing upward FAC area slightly poleward of $68^{\circ}$ latitude. The tilt angle between these two FAC areas is in good agreement with the tilt angle of the flux rope mapped to the ionosphere. The electric field in the region is consistent with a current flow 
from the downward to the upward FAC area in southwestward direction. This is the same direction in which the current in the flux rope is flowing if it is assumed to be close to force-free.

- No ionospheric topological correspondence can be found to the fast $\left(\sim 1200 \mathrm{~km} \mathrm{~s}^{-1}\right)$ earthward movement of the $\mathrm{B}_{X}$ peaks in the magnetosphere related to the TCR.

We suggest a possible interpretation of these topological correspondences as follows: the two FAC regions of opposite polarity may correspond to the ends of the flux rope in the direction of its central axis, where the magnetic topology is not closed and current can partly be diverted into FAC (or vice versa). If this hypothesis applies, it would mean that the current generated further downtail in the magnetosphere can either close via the ionosphere, or "tunnel" through the flux rope in the same direction. Such a current closure during a substorm would only be possible if the core magnetic field in the flux rope points westwards, i.e., in $+Y$ direction like in our case. We emphasize that at the present stage this interpretation is a hypothesis, and more work needs to be done in order to either verify or falsify it. However, the interpretation appears to be consistent with the magnetospheric and ionospheric data and analysis presented in this study.

Finally, we note that while in this paper the Cluster magnetic field and plasma signatures have been interpreted as flux rope and TCR features associated with formation of multiple X-lines, similar magnetic features (bipolar $B_{Z}$ ) were also interpreted as a transient profile of a remote $\mathrm{X}$-line due to its temporal change in the reconnection rate (e.g., Sergeev et al., 2005). The exact discrimination between these two possibilities would require a larger-scale determination of the magnetic field topology, which is beyond of the scope of this study. Yet, the clear associated signatures observed in the ionosphere indicate that in both cases the coupling to the ionosphere would be realised via the current topology determined in this paper.

Acknowledgements. The authors would like to thank J. Slavin for valuable discussions. We are indebted to the director and staff of EISCAT for operating the facility and supplying the data. EISCAT is an International Association supported by Finland (SA), France (CNRS), Germany (MPG), Japan (NIPR), Norway (NFR), Sweden (NFR) and the United Kingdom (PPARC). We like to thank P. Escoubet and the Cluster teams for the successful mission, and H. U. Eichelberger and the Cluster Data Centers for their help in data processing.

Topical Editor W. Kofman thanks T. Nagai and another referee for their help in evaluating this paper.

\section{References}

Amm, O. and Viljanen, A.: Ionospheric disturbance magnetic field continuation from the ground to the ionosphere using spherical elementary current systems, Earth, Planets and Space, 51, 431440, 1999.

Amm, O.: Ionospheric elementary current systems in spherical coordinates and their application, J. Geomagn. Geoelectr., 49, 947955, 1997.

Angelopoulos, V., Baumjohann, W., Kennel, C. F., Coroniti, F. V., Kivelson, M. G., Pellat, R. J., Walker, R. J., Lühr, H., and Paschmann, G.: Bursty bulk flows in the inner central plasma sheet, J. Geophys. Res., 97, 4027-4039, 1992.

Baker, D. N., Pulkkinen, T. I., Angelopoulos, V., Baumjohann, W., and McPherron, R. L.: Neutral line model of substorms, Past results and present view, J. Geophys. Res., 101, 12 975-13010, 1996.

Balogh, A., Carr, C. M., Acuña, M. H., Dunlop, M. W., Beek, T. J., Brown, P., Fornaçon, K. H., Georgescu, E., Glassmeier, K.H., Harris, J., Musmann, G., Oddy, T., and Schwingenschuh, K.: The Cluster Magnetic Field Investigation: overview of inflight performance and initial results, Ann. Geophys., 19, 1207-1217, 2001.

Baumjohann, W., Paschmann, G., and Lühr, H.: Characteristics of high speed ion flows in the plasma sheet, J. Geophys. Res., 95, 3801-3809, 1990.

Dunlop, M. W., Balogh, A., Glassmeier, K.H., and Robert, P.: Fourpoint Cluster application of magnetic field analysis tools: The Curlometer, J. Geophys. Res., 107(A11), 1384, doi:10.1029/2001JA005088, 2002.

Escoubet, C. P., Fehringer, M., and Goldstein, M.: The Cluster mission, Ann. Geophys., 19, 1197-1200, 2001.

Folkestad, K., Hagfors, T., and Westerlund, S.: EISCAT: An updated description of technical characteristics, Radio Sci., 18, 867-879, 1983.

Hones Jr., E. W.: Substorm processes in the magnetotail: Comments on "On hot tenous plasma, fireballs, and boundary layers in the Earth's magneotail” by Frank, L. A., Ackerson, K. L., and Lepping, R. P., J. Geophys. Res., 82, 5633-5643, 1977.

Ieda, A., Machida, S., Mukai, T., Saito, Y., Yamamoto, T., Nishida, A., Terasawa, T., and Kokubun, S.: Statistical analysis of plasmoid evolution with GEOTAIL observations, J. Geophys. Res., 103, 4435-4466, 1998.

Kubyshkina, M. V., Sergeev, V. A., and Pulkkinen, T. I.: Hybrid Input Algorithm: An event-oriented magnetospheric model, J. Geophys. Res., 104, 24 977-24 994, 1999.

Lee, L. C.: A review of magnetic reconnection: MHD models, in: Physics of the magnetopause, edited by: Song, P., Sonnerup, B. U. O., and Thomsen, M. F., AGU Monograph Series, 90, 139$153,1995$.

Lundquist, S.: Magnetohydrostatic fields, Ark. Fys., 2, 361-365, 1950.

Mende, S. B., Heetderks, H., Frey, H. U., Lampton, M., Geller, S. P., Abiad, R., Siegmund, O., Tremsin, A. S., Spann, J., Dougani, H., Fuselier, S. A., Magoncelli, A. L., Bumala, M. B., Murphree, S., and Trondsen, T.: Far ultraviolet imaging from the IMAGE spacecraft: 2. Wideband FUV imaging, Space Sci. Rev., 91, 271285, 2000.

Moldwin, M. B. and Hughes, W. J.: Observations of earthward and tailward propagating flux rope plasmoids: Expanding the plasmoid model of geomagnetic substorms, J. Geophys. Res., 99, 183-198, 1994.

Nagai, T., Fujimoto, M., Saito, Y., Machida, S., Terasawa, T., Naka- 
mura, R., Yamamoto, T., Mukai, T., Nishida, A., and Kokubun, S.: Structure and dynamics of magnetic reconnection for substorm onsets with GEOTAIL observations, J. Geophys. Res., 103, 4419-4440, 1998.

Priest, E. R.: The equilibrium of magnetic flux ropes, Geophys. Monogr., 58, 1-22, 1990.

Rème, H., Aoustin, C., Bosqued, J. M., Dandouras, I., Lavraud, B., Sauvaud, J. A., Barthe, A., Bouyssou, J., Camus, Th., CoeurJoly, O., Cros, A., Cuvilo, J., Ducay, F., Garbarowitz, Y., Medale, J. L., Penou, E., Perrier, H., Romefort, D., Rouzaud, J., Vallat, C., Alcaydé, D., Jacquey, C., Mazelle, C., d'Uston, C., Möbius, E., Kistler, L. M., Crocker, K., Granoff, M., Mouikis, C., Popecki, M., Vosbury, M., Klecker, B., Hovestadt, D., Kucharek, H., Kuenneth, E., Paschmann, G., Scholer, M., Sckopke, N., Seidenschwang, E., Carlson, C. W., Curtis, D. W., Ingraham, C., Lin, R. P., McFadden, J. P., Parks, G. K., Phan, T., Formisano, V., Amata, E., BavassanoCattaneo, M. B., Baldetti, P., Bruno, R., Chionchio, G., Di Lellis, A., Marcucci, M. F., Pallocchia, G., Korth, A., Daly, P. W., Graeve, B., Rosenbauer, H., Vasyliunas, V., McCarthy, M., Wilber, M., Eliasson, L., Lundin, R., Olsen, S., Shelley, E. G., Fuselier, S., Ghielmetti, A. G., Lennartsson, Escoubet, C. P., Balsiger, H., Friedel, R., Cao, J.B., Kovrazhkin, R. A., Papamastorakis, I., Pellat, R., Scudder, J., and Sonnerup, B.: First multispacecraft ion measurements in and near the Earth's magnetosphere with the identical Cluster ion spectrometry (CIS) experiment, Ann. Geophys., 19, 1303-1354, 2001.

Schindler, K.: A theory of the substorm mechanism, J. Geophys. Res., 79, 2803-2810, 1974.

Sergeev, V. A., Kubyshkina, M. V., Baumjohann, W., Nakamura, R., Amm, O., Pulkkinen, T., Angelopoulos, V., Mende, S. B., Klecker, B., Nagai, T., Sauvaud, J.-A., Slavin, J. A., and Thomsen, M. F.: Transition from substorm growth to substorm expansion phase as observed with radial configuration of ISTP and Cluster spacecraft, Ann. Geophys., 23, 2183-2198, 2005.
Sibeck, D. G., Siscoe, G. L., Slavin, J. A., Smith, E. J., Bame, S. J., and Scarf, L.: Magnetotail flux ropes, Geophys. Res. Lett., 11, 1090-1093, 1984.

Slavin, J. A., Fairfield, D. H., Kuznetsova, M. M., Owen, C. J., Lepping, R. P., Taguchi, S., Mukai, T., Saito, Y., Yamamoto, T., Kokubun, S., Lui, A. T. Y., and Reeves, G. D.: ISTP observations of plasmoid ejection: IMP 8 and Geotail, J. Geophys. Res., 103, 119-134, 1998.

Slavin, J. A., Lepping, R. P., Gjerloev, J., Fairfield, D. H., Hesse, M., Owen, C. J., Moldwin, M. B., Nagai, T., Ieda, A., and Mukai, T.: Geotail observations of magnetic flux ropes in the plasma sheet, J. Geophys. Res., 108, 1015, doi:10.1029/2002JA009557, 2003.

Slavin, J. A., Smith, E. J., Tsurutani, B. T., Sibeck, D. G., Singer, H. J., Baker, D. N., Gosling, J. T., Hones, E. W., and Scarf, F. L.: Substorm associated traveling compression regions in the distant tail: ISEE-3 geotail observations, Geophys. Res. Lett., 11, 657660, 1984.

Slavin, J. A., Tanskanen, E., Hesse, M., Owen, C. J., Dunlop, M. W., Imber, S., Lucek, E., Balogh, A., and Glassmeier, K.-H.: Cluster observations of traveling compression regions in the near tail, J. Geophys. Res., 110, A06207, doi:10.1029/2004JA010878, 2005.

Tsyganenko, N. A.: A magnetospheric field model with a warped tail current sheet, Planet. Space Sci., 37, 5-20, 1989.

Untiedt, J. and Baumjohann, W.: Studies of polar current systems using the IMS Scandinavian magnetometer array, Space Sci. Rev., 63, 245-390, 1993.

Vanhamäki, H., Amm, O., and Viljanen, A.: 1dimensional upward continuation of the ground magnetic field disturbance using spherical elementary current systems, Earth Planets Space, 55, 613-625, 2003.

Viljanen, A. and Häkkinen, L.: IMAGE magnetometer network, in: Satellite-Ground Based Coordination Sourcebook, edited by: Lockwood, M., Wild, M. N., and Opgenoorth, H. J., ESA publications SP1198, 111-117, 1997. 